Pacific Journal of Mathematics

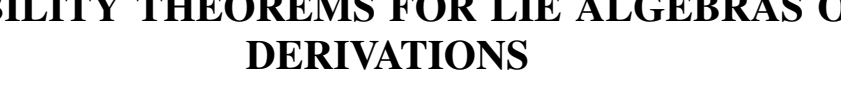




\title{
STABILITY THEOREMS FOR LIE ALGEBRAS OF DERIVATIONS
}

\author{
Charles B. Hallahan
}

\begin{abstract}
Let $A$ be a finite dimensional algebra over a field $F$ of characteristic zero and let $L$ be a completely reducible Lie algebra of derivations of $A$. If $A$ is associative, then there exists an $L$-invariant Wedderburn factor of $A$. If $A$ is a Lie algebra, there exists an $L$-invariant Levi factor of $A$. If $A$ is a solvable Lie algebra, there exists an $L$-invariant Cartan subalgebra of $A$. This paper deals with the uniqueness of such $L$-invariant subalgebras. For the associative case the assumption of characteristic zero can be dropped if we assume that the radical of $A$ is $L$-invariant.
\end{abstract}

2. Preliminaries. If $A$ is a finite dimensional associative algebra over a field $F$ with radical $R$ such that $A / R$ is separable (that is, semisimple and remains so under every field extension of $F$ ), then the Wedderburn principal theorem states that there exists a separable subalgebra $S$ such that $A=S+R, S \cap R=\{0\} . \quad S$ is called a Wedderburn factor of $A$. Since $R$ is nilpotent, for $r$ in $R,(1-r)^{-1}=$ $1+r+\cdots+r^{n-1}$, where $r^{n}=0$. Let $C_{1-r}$ be the inner automorphism of $A$ defined by conjugation by the invertible element $1-r$. The Malcev Theorem states that if $S$ is any separable subalgebra of $A$ and $T$ is a Wedderburn factor of $A$, then there exists $r$ in $R$ such that $C_{1-r}(S) \subseteq T$. Thus, the Wedderburn factors of $A$ are just the maximal separable subalgebras. See [4] for the above information. In $\S 3$ it is shown that if $L$ is completely reducible (every $L$-invariant subspace of $A$ has a complementary $L$-invariant subspace), $F$ arbitrary, $R L$ invariant, and $S, T$ two $L$-invariant Wedderburn factors of $A$, then there exists an element $r$ in $R$ such that $C_{1-r}(S)=T$ and $D(r)=0$ for all $D$ in $L$. Such an element $r$ is called an $L$-constant.

If $A$ is a Lie algebra over a field $F$ of characteristic zero and $R$ is the radical (maximal solvable ideal) of $A$, then the Levi theorem states that $A=S+R, S \cap R=\{0\}$, where $S$ is a semisimple subalgebra of $A$ isomorphic to $A / R$. $S$ is called a Levi factor of $A$. The MalcevHanish-Chandra theorem states that any two Levi factors of $A$ are conjugate by an automorphism $\exp (A d x)$, where $x$ is in $N$, the nil radical (maximal nilpotent ideal) of $A$. In $\S 4$ it is shown that for $L$ completely reducible and $S, T L$-invariant Levi factors of $A$, then there is an $L$-constant $x$ in $N$ such that $\exp (A d x)(S)=T$.

If $A$ is a solvable Lie algebra over a field $F$ of characteristic zero, then any two Cartan subalgebras are conjugate by an automorphism 
of the form $\exp (A d x)$, for $x \in A^{\infty}=\bigcap_{n=1}^{\infty} A^{n}$, see [2]. In $\S 5$, we show that for $L$ completely reducible and $S, T L$-invariant Cartan subalgebras of $A$, then there is a $L$-constant $x$ in $A^{\infty}$ such that $\exp (A d x)(S)=T$.

In [8] Mostow considered the situation where $G$, a completely reducible group of algebra automorphisms, acts on a finite dimensional algebra $A$ over a field $F$ of characteristic zero. For each of the three cases for $A$ mentioned above, Mostow shows that there exists the corresponding kind of $G$-invariant subalgebra. One can use an algebraic group argument, see [1], to conclude the corresponding existence of $L$-invariant subalgebras. The problem of relating $G$-invariant subalgebras has been studied by Taft [9], and uniqueness in that case is given via automorphisms defined by fixed points of $G$. The uniqueness results for $L$-invariant subalgebras (in terms of $L$-constants) can be shown directly, and also, for characteristic zero, can be shown to follow from the results of Taft. It should be noted that if $x$ is an $L$-constant ( $G$-fixed) then $C_{1-x}$ centralizes $L$ (or $G$ ) so that if $S$ is an $L$ (or $G$ ) invariant subalgebra, so is $C_{1-x}(S)$.

Let $F$ have characteristic zero. The relationship between the situations of $L$ acting on $A$ and that of $G$ acting on $A$ is given by the correspondence between a linear algebraic group and its associated Lie algebra, see Chevalley [3]. In particular, if $G$ is an algebraic group of algebra automorphisms of $A$, then its associated Lie algebra will consist of derivations of $A$. Also, complete reducibility is preserved in the algebraic group-Lie algebra correspondence. The following lemma follows easily from the definition of the Lie algebra of an algebraic group. We state it for reference.

Lemma 2.1. Let $V$ be a finite dimensional vector space over a field $F$. Let $G$ be an algebraic group of automorphisms of $V$ and $g$ its associated Lie algebra. If $x$ in $V$ is a fixed point of $G$, then $X(x)=0$ for all $X$ in $g$.

The author would like to express his appreciation to Professor Earl Taft who suggested these problems and acted as thesis advisor during the preparation of this material.

\section{The associative algebra case.}

THEOREM 3.1. Let $A$ be a finite dimensional associative algebra over a field $F$ of characteristic zero and let $L$ be a completely reducible Lie algebra of derivations of $A$. If $S$ is an L-invariant semisimple subalgebra of $A$ and $T$ an L-invariant maximal semisimple subalgebra of $A$, then there exists an L-constant $r$ in $R$, the ra- 
dical of $A$, such that $C_{1-r}$ carries $S$ into $T$.

Proof. Given $L$, let $\bar{L}$ be its algebraic hull, i.e., the smallest algebraic Lie algebra containing $L$, and let $G$ be the unique connected algebraic group of algebra automorphisms with Lie algebra $I$. Then $G$ is also completely reducible. We can apply Theorem 2 of Taft [9] to get $r$ in $R$ such that $C_{1-r}(S) \subseteq T$ and $r$ is a fixed point of $G$. By Lemma 2.1 we have that $X(r)=0$ for all $X$ in $\bar{L}$, and $L \subseteq \bar{L}$ implies that $r$ is an $L$-constant.

CoRollary 1. Let $A$ and $L$ be as in Theorem 3.1. Then any two L-invariant Wedderburn factors of $A$ are conjugate under an inner automorphism of the form $C_{1-r}$, where $r$ is an L-constant in R. Also, we may write $C_{1-r}$ in the form $\exp ($ Ady), where $y$ is an $L$-constant in $R$.

Proof. The first statement follows immediately from Theorem 3.1. Let $y=\log (1-r)=-r-r^{2} / 2-r^{3} / 3-\cdots$. Then $X(y)=0$ for all $x \in L$ and $C_{1-r}=C_{\text {exp }(10 g(1-r))}=\exp (A d(\log (1-r)))=\exp (A d y)$.

Corollary 2. Let $A$ and $L$ be as in Theorem 3.1. Then any $L$-invariant semisimple subalgebra $S$ of $A$ is contained in an $L$ invariant Wedderburn factor.

Proof. Let $T$ be any $L$-invariant Wedderburn factor. By Theorem 3.1 there exists an $L$-constant $r$ in $R$ such that $C_{1-r}(S) \subseteq T$. Thus, $S \subseteq\left(C_{1-r}\right)^{-1}(T)=C_{1-y}(T)$, where $y=-r-r^{2}-r^{3}-\cdots$. Thus $y$ is an $L$-constant in $R$. If $t \in T$, then $C_{1-y}(t)=\left(1+y+\cdots+y^{n}\right) t(1-y)$, where $y^{n+1}=0$. For $D$ in $L, D C_{1-y}(t)=C_{1-y}(D(t))$ since $y$ is an $L$ constant. Thus, $C_{1-y}(T)$ is $L$-invariant.

If we drop the assumption of characteristic zero in Theorem 3.1, then the uniqueness result can be proven directly with the additional hypothesis that $R$ be $L$-invariant. (This is always true for characteristic zero.) The technique used in Theorem 3.1 whereby the situation involving derivations of $A$ is carried over to the situation involving algebra automorphisms of $A$ does not, in general, carry over to the case when $F$ has characteristic $p \neq 0$. It is possible to have an algebraic Lie algebra of derivations of a finite dimensional associative algebra $A$ over a field $F$ of characteristic $p>0$ which is not the Lie algebra of an algebraic group of algebra automorphisms of $A$. This cannot occur in characteristic zero. For example, let $G$ be a cyclic group of order $p$ and $F$ an algebraically closed field of characteristic $p$. Let $A=F(G)$, the group algebra of $G$ over $F$. Then $\left\{1, g, \cdots, g^{p-1}\right\}$ is a basis for $A$ over $F$ and $\left\{g-1, \cdots, g^{p-1}-1\right\}$ is a basis for the 
radical $R$ of $A$. Define a map $D$ of $A$ by $D: g \rightarrow 1$ and extend $D$ to a derivation of $A$. The smallest restricted Lie algebra $L$ of linear transformations of $A$ containing $D$ is algebraic, see [5]. Since the Lie algebra of all derivations of $A$ is restricted, $L$ consists of derivations of $A$. If $G$ is any algebraic group of automorphisms of $A$ with Lie algebra $L$, then $G$ cannot consist of algebra automorphisms of $A$. If so, then $R$ would be $G$-invariant, and, hence, $L$-invariant, which is not the case.

THEOREM 3.2. Let $A$ be a finite dimensional associative algebra over a field $F$ of arbitrary characteristic. Let $R$ be the radical of $A$ and assume $A / R$ is separable. Let $L$ be a completely reducible Lie algebra of derivations of $A$ and assume $R$ is L-invariant. If $S$ is an $L$-invariant separable subalgebra of $A$ and $T$ is an $L$-invariant Wedderburn factor of $A$, then there exists an L-constant $x$ in $R$ such that $C_{1-x}$ carries $S$ into $T$.

Proof. We consider two cases:

Case 1. $R^{2}=\{0\}$. Let $z$ in $R$ be such that $C_{1-z}(S) \subseteq T$. $z$ exists by the Malcev theorem. We claim that $D(z) \in R \cap C$, for all $D \in L$, where $C$ is the centralizer of $S$ in $A$. Given $D \in L$, define $\operatorname{AdD}(z)$, a linear map of $A$, by $A d D(z): a \rightarrow D(z) a-a D(z)$, for $a \in A$. Using the facts that $R^{2}=\{0\}$ and $R$ is $L$-invariant, we have that

$$
A d D(z)=D C_{1-z}-C_{1-z} D .
$$

For $s \in S, A d D(z)(s)=D C_{1-z}(s)-C_{1-z} D(s) \in T$ since $S$ and $T$ are $L$ invariant and $C_{1-\hat{\alpha}}(S) \subseteq T$. By assumption, $D(z) \in R$, so $A d D(z)(S) \in R$. Hence, $\operatorname{AdD}(z): S \rightarrow T \cap R=\{0\}$. Thus, $D(z) \in R \cap C$. $R \cap C$ is an $L$ invariant subspace of $R$, so by complete reducibility we have $R=$ $(R \cap C) \oplus U$, where $U$ is an $L$-invariant subspace of $R$. Write $z=$ $y+x$, where $y \in R \cap C$ and $x \in U$. Thus $x=z-y$ and for $D \in L$, $D(x)=D(z)-D(y) \in(R \cap C) \cap U=\{0\}$. Hence, $x$ is an $L$-constant, and $x=z-y$ where $y \in C$ implies that $C_{1-x}(S)=C_{1-z}(S) \subseteq T$.

If $R^{2} \neq\{0\}$, we proceed by induction on the dimension of $A$. Since $R$ is $L$-invariant, we have that $L$ is a completely reducible Lie algebra of derivations of $R, T+R^{2}$, and $A / R^{2}$, all of which have dimension less than that of $A$. Let $a \rightarrow \bar{a}=a+R^{2}$ denote the natural homomorphism of $A$ onto $\bar{A}=A / R^{2}$. Then $\bar{A}$ has radical $\bar{R}$ and $\bar{S}$ is an $L$ invariant separable subalgebra of $\bar{A}$ while $\bar{T}$ is an $L$-invariant Wedderburn factor of $\bar{A}$. By induction, there exists $\bar{v} \in \bar{R}$ such that $C_{\overrightarrow{1-v}}(\bar{S}) \subseteq \bar{T}$ and $D(v) \in R^{2}$ for all $D$ in $L . R^{2}$ is an $I$-invariant subspace of $R$, so by complete reducibility, we have $R=R^{2} \oplus U$, where $U$ is $L$-invariant. Let $v=z+u, z \in R^{2}, u \in U$. Then $u$ is an $L$-constant and $\bar{u}=\bar{v}$. Consider the algebra $T+R^{2}$. It has dimension less than 
that of $A$, has radical $R^{2}, C_{1-u}(S)$ is an $L$-invariant separable subalgebra of it (since $u$ is an $L$-constant and $S$ is $L$-invariant) and $T$ is an $L$ invariant Wedderburn factor of $T+R^{2}$. By induction, there exists $r$ in $R^{2}$ such that $D(r)=0$ for all $D \in L$ and $C_{1-r} C_{1-u}(S) \subseteq T$. Let $x=$ $u+r-u r$. Then for $D \in L, D(x)=D(u)+D(r)-D(u) r-u D(r)=0$. So $x$ is an $L$-constant and $C_{1-x}(S)=C_{1-r} C_{1-u}(S) \subseteq T$.

Corollary. Let $A$ and $L$ be as in Theorem 3.2. Then every $L$-invariant separable subalgebra of $A$ is contained in an $L$-invariant Wedderburn factor of $A$.

The assumption that $R$ be $L$-invariant is needed in the above theorem. An example can be given of a semisimple derivation $D$ of an associative algebra $A$ over a field of characteristic 3 such that $D$ leaves invariant more than one Wedderburn factor of $A$ and $D(r)=0$ for $r \in R$, the radical of $A$, implies that $r=0$. Let $F$ be any field of characteristic 3 containing roots of the polynomial $x^{3}+x+1$. Let $G$ be a cyclic group of order $3, G=\langle g\rangle, g^{3}=1$, and form the group algebra $F(G)$ of $G$ over $F$. Let $Q$ be the quaternion algebra over $F$, i.e., $Q$ has basis $\{1, i, j, k\}$ over $F$ and $i^{2}=j^{2}=k^{2}=-1$, and $i j=$ $k=-j i, j k=i=-k j, k i=j=-i k$. Let $A=F(G) \otimes_{F} Q$. Then $A$ is an associative algebra over $F$ of dimension 12. A can also be thought of as the algebra of $2 \times 2$ - matrices with entries from $F(G)$. If we write for example, $g i$ for the element $g \otimes i$ of $A$, then $A$ has basis $\left\{1, g 1, g^{2} 1, i, g i, g^{2} i, j, g j, g^{2} j, k, g k, g^{2} k\right\}$. $\{1, i, j, k\}$ forms a basis for a Wedderburn factor $W$ of $A$ and $\left\{g 1-1, g^{2} 1-1, g i-i, g^{2} i-i, g j-j\right.$, $\left.g^{2} j-j, g k-k, g^{2} k-k\right\}$ forms a basis for the radical $R$ of $A$. Then $R^{3}=\{0\}$. Let $r \in R$ where $r=\alpha(g 1-1)+\beta\left(g^{2} 1-1\right)+\gamma\left(g^{2} k-k\right)$ and $\beta \gamma-\alpha \gamma=\gamma-1, \alpha, \beta, \gamma \in F$. Consider the Wedderburn factors of $A$ obtained by applying $C_{1-r}$ to $W$. We get the following bases for the resulting Wedderburn factors:

$$
\begin{aligned}
\left\{1,\left(1+\gamma^{2}\right) i\right. & +\gamma^{2} g i+\gamma^{2} g^{2} i+j+(1-\gamma) g j \\
& +(1+\gamma) g^{2} j,-i+(\gamma-1) g i+(-\gamma-1) g^{2} i \\
& \left.+\left(1+\gamma^{2}\right) j+\gamma^{2} g j+\gamma^{2} g^{2} j, k\right\}=\left\{1, b_{1}, b_{2}, k\right\} .
\end{aligned}
$$

The polynomial $X^{3}+X+1$ has three distinct roots in $F$ and for each distinct root $\gamma$ we define a distinct Wedderburn factor of $A$ by the above. Define a map $D$ of $A$ as follows:

$$
\begin{aligned}
D(1) & =0, D(g 1)=g 1, D\left(g^{2} 1\right)=-g^{2} 1, D(i)=g j, \\
D(g i) & =g i+g^{2} j, D\left(g^{2} i\right)=-g^{2} i+j, D(j)=-g i, \\
D(g j) & =-g^{2} i+g j, D\left(g^{2} j\right)=-i-g^{2} j, D(k)=0, \\
D(g k) & =g k, D\left(g^{2} k\right)=-g^{2} k
\end{aligned}
$$


and extend linearly to all of $A$. Then $D$ defines a derivation of $A$, and it is easy to check that for $r \in R, D(r)=0$ implies that $r=0$. Also, $R$ is not $D$-invariant since $D(g 1-1)=g 1$ and $(g 1)^{3}=1 \notin R$. Also $D$ is semisimple. Consider the Wedderburn factors with bases $\left\{1, b_{1}, b_{2}, k\right\}$ obtained before, where $\gamma^{3}+\gamma+1=0$. Then a direct check shows that $D\left(b_{1}\right)=(\gamma+1) b_{2}$ ahd $D\left(b_{2}\right)=-(\gamma+1) b_{1}$. So all three Wedderburn factors of $A$ are $D$-invariant, and they cannot be conjugate by a $D$-constant in $R$ since the only such constant is 0 .

\section{The Lie algebra case.}

THEOREM 4.1. Let $A$ be a finite dimensional Lie algebra over a field of characteristic zero and $N$ its nil radical. Let $L$ be a completely reducible Lie algebra of derivations of $A$. If $S$ is an $L$ invariant semisimple subalgebra of $A$ and $T$ is an L-invariant Levi factor of $A$, then there exists an L-constant $x$ in $N$ such that $\exp (A d x)$ carries $S$ into $T$.

Proof. The proof is similar to that of Theorem 3.2, and the theorem also follows by using Lemma 2.1 and Theorem 4 of [9], where uniqueness is given in this situation in terms of fixed points of a group of automorphisms of $A$.

\section{Solvable Lie algebras.}

Theorem 5.1. Let $A$ be a finite dimensional solvable Lie algebra over a field of characteristic zero. Let $L$ be a completely reducible Lie algebra of derivations of $A$. If $S$ and $T$ are L-invariant Cartan subalgebras of $A$, then there exists $x$ in $A^{\infty}$ such that $x$ is an L-constant, and $\exp (A d x)(S)=T$.

Proof. An analogous proof to the theorem for groups in [9] can be given. Also the result follows by Lemma 2.1 and Theorem 6 of [9].

If $F$ has characteristic $p \neq 0$, there are examples of solvable Lie algebras with Cartan subalgebras of different dimensions. For arbitrary characteristic Winter [10] has shown that if $G$ is a completely reducible group of automorphisms of a solvable Lie algebra $A$ and $G$ has no nonzero fixed points, then $A$ has at most one $G$-invariant Cartan subalgebra. If $L$ is a completely reducible Lie algebra of derivations of a solvable Lie algebra $A$ over a field of arbitrary characteristic, then one can adapt Winter's proof to show that if $A$ has no nonzero $L$ constants, then $A$ has at most one $L$-invariant Cartan subalgebra. 
6. A counter-example. Let $A$ be a finite dimensional semisimple Lie algebra over an algebraically closed field of characteristic zero and let $s$ be a semisimple automorphism of $A$. Jacobson [6] shows that there exists an $s$-invariant Cartan subalgebra in this situation. The question arises as to whether or not a uniqueness result holds in the sense dealt with previously, i.e., given two $s$-invariant Cartan subalgebras of $A$, are they conjugate by an automorphism $t$ of $A$ such that $t$ commutes with $s$ ? An example will be given to show that uniqueness in this sense need not hold. Let $A$ and $s$ be as above. Recall that $s$ is an invariant automorphism if it is a product

$$
\exp \left(A d x_{1}\right) \cdots \exp \left(A d x_{m}\right),
$$

where each $A d x_{i}$ is a nilpotent derivation of $A$. By a result in BorelMostow [2] there exists a Cartan subalgebra $H$ of $A$ which is pointwise fixed by $s$ when $s$ is also an invariant automorphism. This follows from the fact that if a regular element is left fixed by $S$, then the Cartan subalgebra it determines is left pointwise fixed. So let $s$ be an invariant automorphism of $A$ such that $H$ is a Cartan subalgebra of $A$ left pointwise fixed by $s$. Given any other $s$-stable Cartan subalgebra $T$ of $A$, if uniqueness held we would have an automorphism $t$ of $A$ such that $t: H \rightarrow T$ and $s t=t s$. Then it follows that $T$ is also pointwise fixed by $s$. However, the following example shows that a semisimple invariant automorphism $s$ of a semisimple Lie algebra $A$ need not leave every $s$-stable Cartan subalgebra pointwise fixed. Let $A$ be the simple Lie algebra of $n \times n$-matrices of trace 0 over an algebraically closed field of characteristic zero. Then $A$ has dimension $n^{2}-1$ with Cartan subalgebras of dimension $n-1$. Let $H$ denote the diagonal matrices of trace 0 . Then $H$ has dimension $n-1$ with basis $X_{i}, 2 \leqq i \leqq n$, where $X_{i}$ has 1 in the $(1,1)$-position and -1 in the $(i, i)$-position with zeros elsewhere. Let $M$ be the invertible $n \times n$ matrix with 1 's in the $(i, i+1)$-position, $1 \leqq i \leqq n-1,1$ in the $(n, 1)$ position, and zero elsewhere. Define an automorphism $s$ of $A$ by $s: N \rightarrow$ $M^{-1} N M$ for $n \in A$. Then $s$ is an invariant automorphism of $A$, Jacobson [7, p. 283]. Since $M^{n}=I, s$ has order at most $n$, and so $s$ is semisimple. Thus by the result of Borel-Mostow we know that there exists a Cartan subalgebra of $A$ left pointwise fixed by $s$. One checks directly that $s$ acts on $H$ as follows: $s\left(X_{i}\right)=X_{i+1}-X_{2}$ for $2 \leqq i \leqq n-1$ and $s\left(X_{n}\right)=-X_{2}$. Thus, $H$ is not pointwise fixed by $s$, and it also follows that $s$ has order exactly $n$. 


\section{REFERENCES}

1. L. Auslander and J. Brezin, Almost algebraic Lie algebras, J. Algebra 8, (1968), 295-313.

2. A. Borel and G. D. Mostow, On semisimple automorphisms of Lie algebras, Ann. of Math. (2) 61 (1955), 389-405.

3. C. Chevalley, Théorie des groups de Lie II, Groups Algébriques, Hermann, Paris, 1951.

4. C. Curtis and I. Reiner, Representation theory of finite groups and associative algebras, Interscience, New York, 1962.

5. J. Dieudonne, Sur les groupes de Lie algébriques sur un corps de charactéristique $p>0$, Rend. Cir. Math. Palermo 1 (1952), 380-402.

6. N. Jacobson, A note on automorphisms of Lie algebras, Pacific J. Math. (1962), 305-315.

7. Lie algebras, Interscience, New York, 1962.

8. G. D. Mostow, Fully reducible subgroups of algebraic groups, Amer. J. Math. 78 (1956), 200-221.

9. E. Taft, Orthogonal conjugacies in associative and Lie algebras, Trans. Amer. Math. Soc. 113 (1964). 18-29.

10. D. Winter, On groups of automorphisms of Lie algebras, J. Algebra 8 (1968), 131142.

Received June 24, 1969. The results of this paper are contained in a portion of the author's Doctoral Dissertation, submitted to the Faculty of the Graduate School of Rutgers University in partial fulfillment of the requirements for the degree of Doctor of Philosophy.

UNIVERSITY OF WISCONSIN 


\section{PACIFIC JOURNAL OF MATHEMATICS}

\section{EDITORS}

\author{
H. SAMELSON \\ Stanford University \\ Stanford, California 94305 \\ Richard Pierce \\ University of Washington \\ Seattle, Washington 98105
}

J. DUGUNDJI

Department of Mathematics

University of Southern California

Los Angeles, California 90007

BASIL GORDON*

University of California

Los Angeles, California 90024

\section{ASSOCIATE EDITORS}
E. F. BECKENBACH
B. H. NeUmanN
F. WOLF
K. YoSHIDA

\section{SUPPORTING INSTITUTIONS}

UNIVERSITY OF BRITISH COLUMBIA CALIFORNIA INSTITUTE OF TECHNOLOGY

UNIVERSITY OF CALIFORNIA MONTANA STATE UNIVERSITY

UNIVERSITY OF NEVADA

NEW MEXICO STATE UNIVERSITY

OREGON STATE UNIVERSITY

UNIVERSITY OF OREGON

OSAKA UNIVERSITY

UNIVERSITY OF SOUTHERN CALIFORNIA
STANFORD UNIVERSITY

UNIVERSITY OF TOKYO

UNIVERSITY OF UTAH

WASHINGTON STATE UNIVERSITY

UNIVERSITY OF WASHINGTON

AMERICAN MATHEMATICAL SOCIETY CHEVRON RESEARCH CORPORATION TRW SYSTEMS

NAVAL WEAPONS CENTER 


\section{Pacific Journal of Mathematics}

\section{Vol. 32, No. $1 \quad$ January, 1970}

Robert Alexander Adams, Compact Sobolev imbeddings for unbounded domains ........................................ 1

Bernhard Amberg, Groups with maximum conditions .................. 9

Tom M. (Mike) Apostol, Möbius functions of order k............... 21

Stefan Bergman, On an initial value problem in the theory of two-dimensional transonic flow patterns ................... 29

Geoffrey David Downs Creede, Concerning semi-stratifiable spaces ...... 47

Edmond Dale Dixon, Matric polynomials which are higher

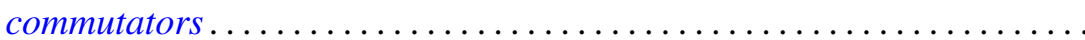

R. L. Duncan, Some continuity properties of the Schnirelmann density.

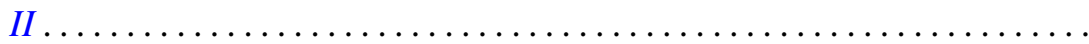

Peter Larkin Duren and Allen Lowell Shields, Coefficient multipliers of $H^{p}$

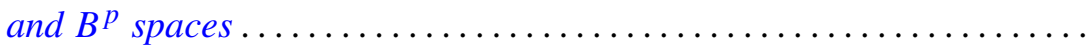

Hector O. Fattorini, On a class of differential equations for vector-valued

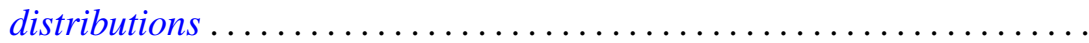

Charles Hallahan, Stability theorems for Lie algebras of derivations. . . . . . 105

Heinz Helfenstein, Local isometries of flat tori ................ 113

Gerald J. Janusz, Some remarks on Clifford's theorem and the Schur

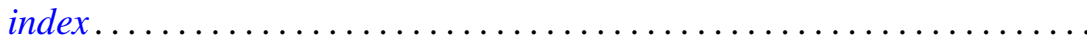

Joe W. Jenkins, Symmetry and nonsymmetry in the group algebras of discrete groups. ...

Herbert Frederick Kreimer, Jr., Outer Galois theory for separable

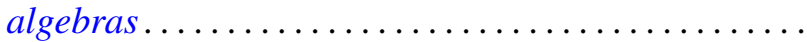

D. G. Larman and P. Mani, On visual hulls

R. Robert Laxton, On groups of linear recurrences. II. Elements of finite order.

Dong Hoon Lee, The adjoint group of Lie groups ...

James B. Lucke, Commutativity in locally compact rings

Charles Harris Scanlon, Rings of functions with certain Lipschitz

$$
\text { properties ............................... }
$$

Binyamin Schwarz, Totally positive differential systems .

James McLean Sloss, The bending of space curves into piecewise helical curves.

James D. Stafney, Analytic interpolation of certain multiplier spaces ...

Patrick Noble Stewart, Semi-simple radical classes.......

Hiroyuki Tachikawa, On left $\mathrm{QF}-3$ rings ...................... 255

Glenn Francis Webb, Product integral representation of time dependent nonlinear evolution equations in Banach spaces.... . . . 